\title{
Prevalence of bone mineral disease among adolescents with cystic fibrosis
}

\author{
Prevalência de doença mineral óssea em adolescentes com fibrose cística \\ Reinaldo José do Amaral Caldeira1, Vânia de Matos Fonseca², \\ Saint Clair dos Santos Gomes Junior ${ }^{3}$, Célia Regina Moutinho de Miranda Chaves ${ }^{4}$
}

\section{Resumo}

Objetivo: Avaliar a prevalência de doença mineral óssea em adolescentes com fibrose cística e associar os achados com as variáveis estudadas.

Métodos: Foram selecionados 37 adolescentes, dos quais foram avaliados: estado nutricional pelos índices de altura/idade e massa corporal/idade; densidade mineral óssea da coluna lombar e corpo inteiro por densitometria com emissão de raio X de dupla energia; ingestão dietética diária pelo registro alimentar de 3 dias; e prova de função pulmonar pelo volume expiratório forçado no primeiro segundo.

Resultados: A média de idade foi de $13,2( \pm 2,8)$ anos. 0 estado nutricional adequado foi de 70,3 e $75,7 \%$ pelos índices de altura/ idade e de massa corporal/idade, respectivamente; $54,1 \%$ dos pacientes apresentaram redução da densidade mineral óssea para coluna lombar e $32,5 \%$ para corpo inteiro. Houve correlação positiva entre densidade mineral óssea e índice de massa corporal $(p=0,04)$. A doença pulmonar e a insuficiência pancreática apresentaram correlação com a alteração da densidade mineral óssea. $O$ inquérito alimentar revelou percentuais de adequação para o cálcio, fósforo e calorias, de acordo com a recomendação nutricional preconizada pelo Consenso Europeu de Fibrose Cística. Essas variáveis não se mostraram estatisticamente significantes na análise multivariada.

Conclusão: A prevalência de doença mineral óssea é alta na adolescência. $O$ estado nutricional adequado, a reposição de enzimas pancreáticas e o controle da doença pulmonar podem ter efeito protetor para a massa óssea.

J Pediatr (Rio J). 2008;84(1):18-25: Densidade óssea, fibrose cística, adolescentes.

\begin{abstract}
Objective: To evaluate the prevalence of bone mineral disease among adolescents with cystic fibrosis and to relate the findings with
\end{abstract} the variables studied.

Methods: The study enrolled 37 adolescents who were assessed for: nutritional status according to height/age and body mass/age ratios; bone mineral density of the lumbar spine and entire body by densitometry with dual emission X-ray; daily dietary intake according to a 3-day dietary recall; and pulmonary function by the forced expiratory volume in one second test.

Results: Mean age was $13.2( \pm 2.8)$ years. Nutritional status was adequate in 70.3 and $75.7 \%$ of patients according to the height/age and body mass/age indices, respectively; $54.1 \%$ of the patients exhibited reduced lumbar spine bone mineral density and $32.5 \%$ for the whole body. There was a positive correlation between bone mineral density and body mass index $(p=0.04)$. Lung disease and pancreatic insufficiency exhibited a correlation with altered bone mineral density. The dietary recall revealed adequate percentages of calcium, phosphorous and calories, according to the nutritional recommendations laid out in the European Cystic Fibrosis Consensus. The multivariate analysis indicated that these variables were not statistically significant.

Conclusions: There is a high prevalence of bone mineral disease among adolescents. Good nutritional status, pancreatic enzyme replacement and control of lung disease may have a protective effect on bone mass.

J Pediatr (Rio J). 2008;84(1):18-25: Bone mineral density, cystic fibrosis, adolescents.

1. Mestre. Instituto Fernandes Figueira, Fundação Oswaldo Cruz (Fiocruz), Rio de Janeiro, RJ.

2. Doutora. Instituto Fernandes Figueira, Fiocruz, Rio de Janeiro, RJ. Instituto de Saúde da Comunidade, Universidade Federal Fluminense (UFF), Rio de Janeiro, RJ.

3. Doutor. Instituto Fernandes Figueira, Fiocruz, Rio de Janeiro, RJ.

4. Doutora. Instituto Fernandes Figueira, Fiocruz, Rio de Janeiro, RJ.

Trabalho vinculado ao Mestrado Profissional do Instituto Fernandes Figueira, Fundação Oswaldo Cruz (Fiocruz), Rio de Janeiro, RJ.

Não foram declarados conflitos de interesse associados à publicação deste artigo.

Como citar este artigo: Caldeira RJ, Fonseca VM, Gomes Jr SC, Chaves CR. Prevalence of bone mineral disease among adolescents with cystic fibrosis. J Pediatr (Rio J). 2008;84(1):18-25.

Artigo submetido em 08.05.07, aceito em 01.08.07.

doi:10.2223/JPED.1737 


\section{Introdução}

A criação de centros de referência em fibrose cística (FC) e o avanço alcançado pela comunidade científica em biologia molecular na genética, transporte iônico, imunologia, fisioterapia respiratória, suplementação nutricional, formulações enzimáticas, antibioticoterapia e transplantes pulmonares permitiram o aumento da expectativa de vida desses pacientes para mais de 30 anos $^{1,2}$. Porém, em conseqüência desta maior longevidade, surgiu uma complicação, a doença mineral óssea (DMO), descrita pela primeira vez em 1979 por Mischler et al. ${ }^{3}$.

Uma vez que a sintomatologia da fragilidade óssea é incomum no final da infância e adolescência e devido ao curso lento e progressivo da doença, a fratura óssea pode ser a primeira manifestação da $\mathrm{FC}^{4}$.

Apesar de a patogênese da DMO relacionada à $\mathrm{FC}$ ainda não estar totalmente esclarecida, acredita-se que ela seja multifatorial ${ }^{5}$ : na má absorção de vitaminas $\mathrm{D}$ e $\mathrm{K}$, encontrada em $40 \%$ dos casos de $\mathrm{FC}^{6}$; na baixa ingestão e absorção de cálcio e desnutrição, pela insuficiência pancreática presente em $85-90 \%$ dos fibrocísticos ${ }^{7}$; no atraso da puberdade/hipogonadismo, encontrado em 73 a $88 \%$ dos casos de $\mathrm{FC}^{7}$; na resposta inflamatória pulmonar, principal responsável por $80 \%$ das mortes por $\mathrm{FC}^{8}$; na diminuição da atividade física e sedentarismo, como principal conseqüência da doença pulmonar supurativa crônica; e na corticoterapia inalada e oral usada em 82 e $31 \%$ dos casos de FC, respectivamente ${ }^{9}$.

A maioria dos pacientes adultos atendidos nos centros de referência para FC apresenta significativa deficiência na mineralização óssea e alto risco de fraturas aos mínimos traumas das vértebras e das costelas ${ }^{7,10}$.

Apesar de estudos com adolescentes fibrocísticos demonstrarem que eles também apresentam osteoporose ou osteopenia, o tratamento medicamentoso da DMO pode apresentar efeitos indesejáveis, como refluxo gastroesofágico e esofagite, além de absorção diminuída na presença de alimen$\operatorname{tos}^{11,12}$. A prevenção é, portanto, o caminho mais efetivo, alcançado pelo controle nutricional e respiratório, prática de atividade física e exposição ao sol na manutenção da saúde óssea e melhora da qualidade de vida desses pacientes ${ }^{7}$.

Assim, neste estudo, buscou-se investigar a prevalência de DMO em adolescentes com FC atendidos no Instituto Fernandes Figueira (IFF) da Fundação Oswaldo Cruz, que é centro de referência do Ministério da Saúde, com o objetivo de contribuir para a sua prevenção.

\section{Métodos \\ Estudo}

Para este estudo, realizou-se um censo com todos os pacientes entre 10 e 18 anos com diagnóstico de FC que foram atendidos no IFF entre maio e junho de 2006. Os critérios de inclusão foram: ter realizado o exame de densitometria óssea
(DO), não ser portador de DMO primária e não ter se submetido a transplantes pulmonares. Os dados foram coletados a partir dos prontuários dos pacientes atendidos nos ambulatórios de pneumologia, nutrição, pediatria e fisioterapia. No período de coleta de dados, 37 pacientes atenderam aos critérios de inclusão. Estes pacientes fazem parte de uma coorte acompanhada sistematicamente pelo IFF desde o momento do diagnóstico da doença. O número de pacientes analisados garante, considerando uma prevalência variando de 40 a 60\% de DMO nos pacientes com FC, confiabilidade de $99 \%$ e poder de $80 \%$ para as análises estatísticas realizadas.

Este estudo utilizou uma base de dados secundários (prontuário de atendimento médico regular), normalmente sujeita a vieses de informação. Isso possivelmente foi minimizado pelo fato de que dois dos autores deste trabalho são responsáveis pelo acompanhamento desses pacientes, realizando grande parte dos registros, com um controle de qualidade facilitado ainda pelo pequeno número de indivíduos doentes. $\mathrm{O}$ viés de memória sobre as variáveis declaradas pelo responsável pela criança (atividade física, exposição ao sol, uso de medicamentos) também pode ter ocorrido. Entretanto, o paciente com FC, pelas próprias características da doença, exige uma relação médico-paciente-cuidador da criança de muita proximidade e confiança, na qual as informações estão muito presentes na memória do cuidador.

\section{Coleta de dados}

Foram coletados dados referentes à identificação do adolescente (nome, número do prontuário, idade e sexo), idade na época do diagnóstico da FC, presença de suficiência ou insuficiência pancreática de acordo com exame de gordura fecal pelo método quantitativo, presença de diabetes melito pelo teste positivo de tolerância oral à glicose e uso de vitamina D e vitamina K. Para avaliação antropométrica, foi utilizado o padrão de curvas de crescimento do National Center for Health Statistics (NCHS) ${ }^{13}$, através do índice de estatura/ idade (E/I) e índice de massa corporal/idade (IMC/I), adotando-se como ponto de corte os referidos pelo Centers for Disease Control and Prevention (CDC), considerado em percentil. A composição corporal foi obtida pela equação de Slaughter ${ }^{14}$, (massa livre de gordura e massa gorda corporal). A avaliação da ingestão de calorias, cálcio e fósforo foi obtida pelo programa NutWin versão $1.5^{15}$. A ingestão calórica foi calculada como porcentagem de recomendação preconizada para esses pacientes e considerada adequada quando atingiu de 20 a 50\% acima do recomendado ${ }^{16,17}$. Atividade física foi considerada como o hábito de praticar exercícios diariamente, no mínimo por 20 minutos, três vezes por semana ${ }^{18}$. Exposição ao sol foi considerada quando ocorria por mais de 30 minutos por dia ${ }^{19}$.

A integridade óssea foi avaliada pela presença ou ausência de fratura e pelo tipo de trauma (leve ou espontâneo e severo). Foram feitas anotações sobre o uso e a via de administração de corticóide, se oral ou inalado, e quanto à presença de osteoporose familiar. 
A prova de função pulmonar foi obtida por espirometria, considerada válida até 6 meses antes da coleta de dados, ressaltando que esses pacientes realizam prova de função pulmonar duas vezes ao ano, utilizando a medida do volume expiratório forçado no primeiro segundo $\left(\operatorname{VEF}_{1}\right)$, que classifica a gravidade dos distúrbios ventilatórios obstrutivos e restritivos em leve ( $\geq 60 \%$ ), moderado (de 41 a $59 \%$ ) e grave $(\leq 40 \%)^{20}$. Foi considerado colonizado o paciente com mais de três culturas de escarro positivas em um intervalo de até 6 meses para bactérias específicas de FC, como Staphylococcus aureus, Pseudomonas aeruginosa e Burkholderia cepacia $^{1}$.

A DO para medida da densidade mineral óssea foi obtida pelo método de absorciometria com emissão de raios $X$ de dupla radiação (DEXA) nas incidências do corpo inteiro e da coluna lombar (L1 a L4), utilizando o aparelho modelo Prodigy Advance, marca Lunar/GE Healthcare (software versão 9.15). Para o diagnóstico da DMO, foi utilizado o escore $z$, segundo critérios da Organização Mundial da Saúde, que considera normal até -1 ; osteopenia de -1 a $-2,5$; e osteoporose abaixo de $-2,5^{21}$.

\section{Análises estatísticas}

Foram utilizados os testes qui-quadrado e Fisher para as variáveis categóricas. Para as variáveis contínuas, foi utilizado o teste $t$ de Student (variáveis com distribuição normal), e o teste não-paramétrico de Kruskal-Wallis para variáveis que não apresentaram distribuição normal. As variáveis com significância estatística na análise bivariada ou clinicamente relacionadas com o desfecho foram incorporadas a um modelo de regressão logística para uma análise multivariada. O método de stepwise foi utilizado para a definição do modelo final. Os testes estatísticos foram realizados tendo como referência um nível de significância $\mathrm{p} \leq 0,05$ para os parâmetros estimados. A análise bivariada foi realizada utilizando o programa Epi-Info versão 3.3.2 ${ }^{22}$. Para a regressão logística, utilizou-se o programa SPSS versão 12 para Windows.

A pesquisa foi aprovada pelo comitê de ética em pesquisa do IFF envolvendo seres humanos (em 31/05/2006).

\section{Resultados}

Foram avaliados 37 adolescentes (Tabela 1) com FC, apresentando mediana de idade de 13 anos. Nesse conjunto, $59,5 \%$ eram do sexo feminino e $40,5 \%$ do masculino. A mediana de idade do paciente na ocasião em que foi feito $o$ diagnóstico de $\mathrm{FC}$ foi de 2 anos.

O exame de DO revelou que, na coluna lombar, a osteopenia foi mais prevalente no sexo feminino $(53,8 \%)$ e a osteoporose no masculino $(57,1 \%)$. No corpo inteiro, a osteopenia também foi mais prevalente no sexo feminino (70\%), mas a osteoporose apresentou o mesmo percentual de prevalência para os dois gêneros. Em seu conjunto, a amostra estudada apresentou $54,1 \%$ de DO alterada e 45,9\% normal.
Os grupos mostraram-se semelhantes entre as diversas variáveis analisadas (Tabelas 2 e 3). As diferenças ocorreram na distribuição: do IMC $(p=0,04)$; da insuficiência pancreática $(p=0,003)$; da colonização bacteriana $(p=0,03)$; da prova de função respiratória $(p=0,03)$; e do uso de vitaminas D e K $(p=0,03)$. Entretanto, essas variáveis não se mostraram significantes na análise de associação realizada pelo modelo de regressão logística.

\section{Discussão}

A mediana de 13 anos da idade cronológica encontrada nos 37 adolescentes deste estudo não é um indicador suficiente do ritmo de crescimento e das necessidades nutricionais, porque existe uma grande variabilidade individual entre os indivíduos nesta faixa etária, quando ocorrem dois eventos importantes quase simultaneamente: o estirão de crescimento, caracterizado por incremento substancial da estatura, e o pico de massa óssea. Neste estudo, não foi observado atraso da puberdade, talvez pela reduzida mediana de idade encontrada.

A mediana de 2 anos de idade na ocasião do diagnóstico de FC dos adolescentes estudados é considerada tardia. Isso pode ser explicado pela FC ser uma doença ainda pouco conhecida e de difícil diagnóstico. Segundo Gibson et al. ${ }^{1}$, a maioria dos casos de FC é identificada com base em sintomas clínicos, dos quais $43,8 \%$ são respiratórios, $24,4 \%$ relativos à deficiência de desenvolvimento e $18,5 \%$ por íleo meconial. O diagnóstico nem sempre é definido pelo teste do suor, pois 1 a $2 \%$ dos pacientes com FC apresenta concentração normal de eletrólitos no suor. Em 71\% dos casos, o diagnóstico é estabelecido em torno de 1 ano, em $8 \%$ após os 10 anos de idade e em $4 \%$ dos casos na vida adulta ${ }^{23,24}$.

O método para mensuração da densidade mineral óssea utilizado neste estudo foi o DEXA, considerado padrão-ouro, que mede tanto o esqueleto axial quanto o apendicular, sendo, portanto, capaz de avaliar o osso cortical e o trabecular. Uma das grandes vantagens do método é o baixo risco de exposição à radiação (30 vezes menor que a empregada no raio $\mathrm{X}$ ). O exame é capaz de detectar perdas inferiores a $5 \%$, ao passo que o raio $X$ detecta perdas da massa óssea a partir de 30 a $50 \%{ }^{11}$.

A prevalência de pacientes que tinham DO alterada foi de $54,1 \%$. Estudo realizado por Bhudhikanok et al. ${ }^{25}$, em 49 crianças, adolescentes e adultos com FC, também encontrou osteopenia em 21 (42,8\%) crianças e adolescentes. No presente estudo, observou-se maior comprometimento da DO da coluna lombar, com 35,1\% de osteopenia e 18,9\% de osteoporose. Isso pode ser explicado pelo fato de aproximadamente $65 \%$ das vértebras serem formadas por ossos trabeculares ${ }^{26}$, que são os mais vulneráveis ao uso de corticóides, às citocinas inflamatórias e à diminuição dos hormônios sexuais estradiol e testosterona, características presentes na FC. Além disso, a alteração da DO no corpo inteiro é menos comum, porque grande parte do esqueleto é formado de osso cortical com mineralização mais resistente e 
Tabela 1 - Distribuição das características dos adolescentes atendidos no IFF, segundo a presença de fatores envolvidos na doença óssea de maio a junho, 2006

\begin{tabular}{|c|c|c|}
\hline Fator & $\mathbf{n}$ & $\%$ \\
\hline \multicolumn{3}{|l|}{ Estado nutricional } \\
\hline Estatura/idade normal $(P>5)$ & 26 & 70,3 \\
\hline Baixa estatura/idade $(P \leq 5)$ & 11 & 29,7 \\
\hline Peso adequado (IMC; P > 5) & 28 & 75,7 \\
\hline Baixo peso (IMC; $P \leq 5)$ & 9 & 24,3 \\
\hline \multicolumn{3}{|l|}{ Colonizado } \\
\hline $\operatorname{Sim}$ & 29 & 78,4 \\
\hline Não & 8 & 21,6 \\
\hline \multicolumn{3}{|l|}{ Prova função respiratória* } \\
\hline $\mathrm{VEF}_{1} \leq 40$ & 3 & 8,3 \\
\hline $\mathrm{VEF}_{1} 41$ a 59 & 6 & 16,7 \\
\hline $\operatorname{VEF}_{1} \geq 60$ & 27 & 75 \\
\hline \multicolumn{3}{|l|}{ Insuficiência pancreática } \\
\hline $\operatorname{Sim}$ & 29 & 78,4 \\
\hline Não & 8 & 21,6 \\
\hline \multicolumn{3}{|l|}{ Diabetes melito } \\
\hline Sim & 4 & 10,8 \\
\hline Não & 33 & 89,2 \\
\hline \multicolumn{3}{|l|}{ Reposição de vitamina D e K } \\
\hline $\operatorname{Sim}$ & 29 & 78,4 \\
\hline Não & 8 & 21,6 \\
\hline \multicolumn{3}{|l|}{ Ingestão de cálcio } \\
\hline Adequada & 20 & 54,1 \\
\hline Inadequada & 17 & 45,9 \\
\hline \multicolumn{3}{|l|}{ Ingestão de fósforo } \\
\hline Adequada & 34 & 91,9 \\
\hline Inadequada & 3 & 8,1 \\
\hline \multicolumn{3}{|l|}{ Ingestão de calorias } \\
\hline Adequada & 34 & 91,9 \\
\hline Inadequada & 3 & 8,1 \\
\hline \multicolumn{3}{|l|}{ Fraturas* } \\
\hline Sim & 12 & 33,3 \\
\hline Não & 24 & 66,7 \\
\hline \multicolumn{3}{|l|}{ Tipo de trauma* } \\
\hline Ausente & 24 & 66,7 \\
\hline Leve ou espontâneo & 9 & 25 \\
\hline Severo & 3 & 8,3 \\
\hline \multicolumn{3}{|l|}{ Corticoterapia $^{+}$} \\
\hline Inalado & 11 & 29,7 \\
\hline Não utiliza & 26 & 70,3 \\
\hline \multicolumn{3}{|l|}{ Osteoporose familiar* } \\
\hline Presente & 9 & 25 \\
\hline Ausente & 27 & 75 \\
\hline \multicolumn{3}{|l|}{ Atividade física } \\
\hline Pratica & 25 & 67,6 \\
\hline Não pratica & 12 & 32,4 \\
\hline \multicolumn{3}{|l|}{ Tempo de exposição ao sol* } \\
\hline$>30 \mathrm{~min}$ & 22 & 61,1 \\
\hline$<30 \mathrm{~min}$ & 14 & 38,9 \\
\hline
\end{tabular}

IMC = índice de massa corporal; $\mathrm{P}=$ percentil; $\mathrm{VEF}_{1}=$ volume expiratório forçado no primeiro segundo.

* Nem todos os pacientes apresentaram informação para esta variável.

+ Não foi apresentada a variável corticoterapia oral porque nenhum paciente fez uso da via oral. 
Tabela 2 - Distribuição das variáveis contínuas segundo o exame de densitometria óssea dos adolescentes com fibrose cística atendidos no Instituto Fernandes Figueira de maio a junho, 2006

\begin{tabular}{|c|c|c|c|c|c|}
\hline \multirow[b]{3}{*}{ Variáveis } & \multicolumn{5}{|c|}{ Densitometria óssea } \\
\hline & \multicolumn{2}{|c|}{ Normal (escore $z \geq-1$ ) $n=17$} & \multicolumn{2}{|c|}{ Alterada $($ escore $z<-1) n=20$} & \multirow[b]{2}{*}{$\mathbf{p}$} \\
\hline & Média (DP) & Mediana & Média (DP) & Mediana & \\
\hline Idade (anos) & $13,7(2,8)$ & 14,0 & $12,7(2,7)$ & 12,0 & 0,30 \\
\hline Idade no diagnóstico (anos) & $3,2(4,7)$ & 1,0 & $5,5(4,9)$ & 5,0 & 0,15 \\
\hline Peso $(\mathrm{kg})$ & $42,3(13,0)$ & 40,0 & $34,8(13,1)$ & 30,0 & 0,08 \\
\hline $\operatorname{IMC}\left(\mathrm{kg} / \mathrm{m}^{2}\right)$ & $18,8(3,4)$ & 17,6 & $16,8(2,4)$ & 15,6 & 0,04 \\
\hline $\operatorname{VEF}_{1}(\%)$ & $87,5(20,1)$ & 93,5 & $69,2(33,5)$ & 71,0 & $0,10 *$ \\
\hline Ingestão calórica (Kcal) & $2.481,8(650,8)$ & $2.450,0$ & $2.651,7(875,2)$ & $2.430,0$ & 0,50 \\
\hline Ingestão de cálcio (mg) & $1.062,1(495,7)$ & 953,7 & $951,6(368,9)$ & 951,1 & 0,44 \\
\hline Ingestão de fósforo (mg) & $1.494(560,2)$ & $1.541,9$ & $1.337,9(639,4)$ & $1.375,9$ & 0,43 \\
\hline Exposição ao sol (min/dia) & $52,3(37,8)$ & 40,0 & $46,6(34,6)$ & 45,5 & 0,62 \\
\hline Massa livre de gordura (kg) & $32,0(8,7)$ & 33,1 & $29,6(10,5)$ & 26,1 & 0,46 \\
\hline Massa gorda corporal (kg) & 9,4 & 8,4 & $6,7(4,7)$ & 5,2 & \\
\hline
\end{tabular}

$\mathrm{DP}$ = desvio padrão; IMC = índice de massa corporal; $\mathrm{VEF}_{1}=$ volume expiratório forçado no primeiro segundo.

* Teste de Kruskal-Wallis.

tecido ósseo compacto, predominando nos ossos longos, colo do fêmur e parte do rádio. Trabalho de Ujhelyi et al. ${ }^{27}$, em 44 pacientes, detectou alta proporção de deformidades na coluna vertebral, afetando quase exclusivamente a estrutura das vértebras torácicas. As manifestações clínicas da osteoporose podem ocorrer muito cedo, já na infância e início da adolescência, e não têm sido valorizadas ${ }^{7}$. Por isso, Kerem et al. ${ }^{17}$ orientam que os pacientes com FC a partir dos 6 anos de idade já devam realizar exame de DO a cada 2 ou 3 anos, ou com maior freqüência na presença de doença pulmonar e uso de corticóide oral. Cabe ressaltar que, após o diagnóstico, a eficácia do tratamento medicamentoso da osteoporose, além de apresentar seus efeitos indesejáveis, é pequena, e o ganho de massa óssea após 2 anos é de apenas 5 a $10 \%$ na coluna lombar, o que aumenta a importância de controlar os fatores predisponentes.

Outro fato relevante é que a osteoporose, segundo Shane et al. ${ }^{28}$, é um fator limitante para o transplante pulmonar, pois o uso de grandes doses de imunossupressores, incluindo corticosteróides e ciclosporinas, e longos períodos de repouso e inatividade pós-transplante podem contribuir para a DMO.

A média do IMC/I foi menor nos adolescentes com DO alterada, o que está de acordo com os resultados de Mischler ${ }^{3}$, que, ao estudar 27 pacientes com FC de 5 a 24 anos, encontrou baixo peso na maioria dos pacientes com desmineralização óssea. Frangolias et al. ${ }^{18}$ relacionaram o IMC/I com a DO e também evidenciaram DMO no quadril e vértebras em $85 \%$ daqueles com redução do IMC/I. Nos adolescentes estudados, $24,3 \%$ dos casos também apresentaram baixo peso pelo
IMC/I. Isso evidencia que a nutrição é crítica no desenvolvimento do adolescente e que o consumo de dieta inadequada pode alterar o crescimento somático, sobretudo do esqueleto, que atinge $90 \%$ de sua maturação até os 18 anos, sendo que pouco pode ser feito após essa idade. Esses achados evidenciam que o estado nutricional está associado à DMO.

O comprometimento pulmonar, evidenciado pela presença de colonização bacteriana em $65 \%$ dos casos na DO alterada, apresentou uma associação positiva com a DMO. Esse resultado está de acordo com Gronowitz et al. ${ }^{29}$, que, ao estudarem a DMO em pacientes com peso e estatura normais, observaram que estava estreitamente relacionada à perda da função respiratória. As categorias dos valores de $\mathrm{VEF}_{1}$ da prova de função respiratória estavam diminuídas na DO alterada. A lesão pulmonar secundária à inflamação tecidual pós-colonização é acompanhada do aumento da concentração de citocinas circulantes e precede a alteração da função pulmonar, e essas citocinas estimulam a reabsorção e inibem a formação óssea, explicando por que a doença pulmonar está associada à DMO.

A maioria da população estudada apresentou os níveis de adequação na ingestão de calorias, cálcio e fósforo acima do recomendado pelo European Cystic Fibrosis Consensus ${ }^{30}$. Porém, a média de ingestão de cálcio $(951,6 \mathrm{mg})$ e fósforo $(1.337,9 \mathrm{mg})$ foi menor nos pacientes com DO alterada. Diante disso, a atenção dos cuidadores deve estar voltada para as grandes exigências de nutrientes e calorias em adolescentes com FC, conseqüente ao crescimento acelerado, nível de gravidade da doença e prática de atividade física. 
Tabela 3 - Distribuição das variáveis categóricas, segundo o exame de densitometria óssea dos adolescentes com fibrose cística atendidos no Instituto Fernandes Figueira de maio a junho, 2006

\begin{tabular}{|c|c|c|c|c|c|}
\hline \multirow[b]{3}{*}{ Variáveis } & \multicolumn{5}{|c|}{ Densitometria óssea } \\
\hline & \multicolumn{2}{|c|}{ Normal (escore $z \geq-1$ ) $n=17$} & \multicolumn{2}{|c|}{ Alterada (escore $z<-1) n=20$} & \multirow[b]{2}{*}{$\mathbf{p}$} \\
\hline & $\mathbf{n}$ & $\%$ & $\mathbf{n}$ & $\%$ & \\
\hline \multicolumn{6}{|l|}{ Sexo } \\
\hline Feminino & 12 & 70,6 & 10 & 50,0 & 0,17 \\
\hline Masculino & 5 & 29,4 & 10 & 50,0 & \\
\hline \multicolumn{6}{|l|}{ Estado nutricional } \\
\hline Estatura/idade normal $(P>5)$ & 14 & 82,4 & 12 & 60,0 & $0,13^{*}$ \\
\hline Baixa estatura/idade $(P \leq 5)$ & 3 & 17,6 & 8 & 40,0 & \\
\hline Peso adequado (IMC; P > 5) & 15 & 88,2 & 13 & 65,0 & $0,10 *$ \\
\hline Baixo peso (IMC; $P \leq 5$ ) & 2 & 11,8 & 7 & 35,0 & \\
\hline \multicolumn{6}{|l|}{ Insuficiência pancreática } \\
\hline Sim & 17 & 100,0 & 12 & 60,0 & $0,003^{+}$ \\
\hline Não & 0 & 0,0 & 8 & 40,0 & \\
\hline \multicolumn{6}{|l|}{ Diabetes melito } \\
\hline Sim & 2 & 11,8 & 2 & 10,0 & $0,63 *$ \\
\hline Não & 15 & 88,2 & 18 & 90,0 & \\
\hline \multicolumn{6}{|l|}{ Colonização bacteriana } \\
\hline Sim & 16 & 94,1 & 13 & 65,0 & $0,03 *$ \\
\hline Não & 1 & 5,9 & 7 & 35,0 & \\
\hline \multicolumn{6}{|l|}{ Prova função respiratória* } \\
\hline $\operatorname{VEF}_{1} \leq 40$ & 16 & 94,1 & 11 & 57,9 & $0,03^{+}$ \\
\hline $\operatorname{VEF}_{1} 41$ a 59 & 1 & 5,9 & 5 & 26,3 & \\
\hline $\operatorname{VEF}_{1} \geq 60$ & 0 & 0,0 & 3 & 15,8 & \\
\hline \multicolumn{6}{|l|}{ Vitamina D e K } \\
\hline $\operatorname{Sim}$ & 17 & 100,0 & 12 & 60,0 & $0,03^{+}$ \\
\hline Não & 0 & 0,0 & 8 & 40,0 & \\
\hline \multicolumn{6}{|l|}{ Fraturas } \\
\hline Sim & 4 & 25,0 & 7 & 35,0 & $0,39 *$ \\
\hline Não & 12 & 75,0 & 13 & 65,0 & \\
\hline \multicolumn{6}{|l|}{ Tipo de trauma } \\
\hline Ausente & 11 & 68,8 & 13 & 65,0 & $0,58 *$ \\
\hline Leve/espontâneo & 3 & 18,8 & 6 & 30,0 & \\
\hline Severo & 2 & 12,5 & 1 & 5,0 & \\
\hline \multicolumn{6}{|l|}{ Osteoporose familiar } \\
\hline Sim & 5 & 31,2 & 4 & 20,0 & $0,34 *$ \\
\hline Não & 11 & 68,8 & 16 & 80,0 & \\
\hline \multicolumn{6}{|l|}{ Exposição ao sol } \\
\hline Sim & 15 & 93,8 & 18 & 90,0 & $0,58 *$ \\
\hline Não & 1 & 6,2 & 2 & 10,0 & \\
\hline \multicolumn{6}{|l|}{ Atividade física } \\
\hline Pratica & 11 & 64,7 & 14 & 70,0 & $0,37 *$ \\
\hline Não Pratica & 6 & 35,3 & 6 & 30,0 & \\
\hline
\end{tabular}

$\mathrm{IMC}=$ índice de massa corporal; $\mathrm{P}=$ percentil; $\mathrm{VEF}_{1}=$ volume expiratório forçado no primeiro segundo.

* Teste de Fisher exato.

+ Não foi possível o teste estatístico devido ao valor zero.

Sessenta por cento dos pacientes com insuficiência pancreática apresentaram DO alterada. Esse resultado deve ser valorizado, uma vez que mais de $90 \%$ dos pacientes com FC apresentam insuficiência pancreática exócrina, estando em risco para o desenvolvimento da DMO devido à má absorção de vitaminas lipossolúveis ( $D$ e $K$ ) e à deficiência nutricional $^{29}$. Um fato que não deve ser esquecido é que a sintomatologia da doença pancreática só se manifesta quando $98 \%$ 
do pâncreas foram destruídos. Por isso, o diagnóstico da doença pancreática deve ser sempre pesquisado para dar início à reposição enzimática e suplementação vitamínico-mineral.

A presença de fratura por trauma leve foi mais freqüente nos pacientes com DO alterada. Esse dado deve ser valorizado, pois a ocorrência deste tipo de fratura durante as atividades da vida diária pode ser o primeiro sinal da osteoporose. Buntain et al. ${ }^{26}$ também observaram alta prevalência de fraturas em adolescentes com FC, principalmente das vértebras. A fratura debilita o paciente com FC, muitas vezes de forma irreversível, e quando ocorre na costela pode alterar a evolução clínica ao causar dor torácica, inibir o reflexo da tosse e remoção de secreções das vias aéreas, acelerando o declínio da função pulmonar.

Cerca de $10 \%$ dos pacientes com DO alterada não foram expostos ao sol e, entre os expostos, o tempo médio foi menor do que naqueles com DO normal. Os trabalhos de Reiter et al. ${ }^{19}$ correlacionaram as estações do ano no hemisfério norte com os níveis de vitamina $D$ no sangue e demonstraram o aumento do risco de osteopenia com a diminuição de vitamina $D$ sérica em função da escassez de luz solar no inverno, mostrando a sua importância na produção de vitamina $D$.

Neste estudo, nenhum paciente utilizou corticoterapia oral. O corticóide inalado foi utilizado tanto por pacientes com DO normal como alterada, sem diferença estatística. BalfourLynn ${ }^{31}$ também observou que a corticoterapia inalada, mesmo em altas doses, possui pouco efeito na densidade óssea e nas fraturas. Entretanto, Bhudhikanok et al. ${ }^{25}$, estudando 21 adolescentes com menos de 18 anos em uso de corticóide oral, concluíram que o uso de corticóide sistêmico é fator de risco para DMO.

Os fatores de risco eleitos foram analisados por um modelo de regressão, porém não se mostram significativamente associados com a DMO. Muito provavelmente, este resultado esteja refletindo o fato de a população estudada ter menos comprometimento com relação a essas variáveis; um exemplo disso é mostrado pelo IMC que, neste grupo, foi compatível com adolescentes eutróficos. O mesmo ocorreu com relação ao $\mathrm{VEF}_{1}$, que apresentou valores altos típicos de doença pulmonar leve ou normalidade.

Apesar da alta prevalência de DMO na população estudada, por tratar-se de um estudo transversal, algumas limitações devem ser consideradas. A mais importante delas é a questão da temporalidade, mostrar se o fator de exposição precede o desfecho estudado. Estudos longitudinais com acompanhamento sistemático destes pacientes poderiam auxiliar no entendimento da história natural da DMO e, conseqüentemente, nas estratégias de prevenção e tratamento destes pacientes. Esses estudos incluiriam medidas como ingestão de calorias e cálcio, exposição ao sol, prática de atividade física, uso regular de enzimas e vitaminas lipossolúveis $\mathrm{D}$ e K, controle adequado da infecção pulmonar e exames periódicos de DO a partir de 10 anos de idade. Portanto, devido à escassez de informações sobre o assunto na população de pacientes com FC atendida no Brasil, recomendamos a realização de estudos prospectivos.

Concluímos que existe uma alta prevalência de DMO na população estudada de adolescentes com $\mathrm{FC}$, sendo a coluna lombar mais freqüente. A doença pulmonar, a insuficiência pancreática e a desnutrição foram os fatores de risco mais estreitamente associados com a DMO. Todos os esforços de prevenção devem ser direcionados para o final da infância e início da adolescência, uma vez que, caso a osteoporose não seja diagnosticada no adolescente com FC, após esse período pouco poderá ser feito para aumentar a massa óssea.

\section{Referências}

1. Gibson RL, Burns JL, Ramsey BW. Pathophysiology and management of pulmonary infections in cystic fibrosis. Am J Respir Crit Care Med. 2003;168:918-51.

2. Botton E, Saraux A, Laselve H, Jousse S, Le Goff. Musculoskeletal manifestations in cystic fibrosis. Joint Bone Spine. 2003;70:32735.

3. Mischler EH, Chesney PJ, Chesney RW, Mazess RB. Demineralization in cystic fibrosis detected by direct photon absorptiometry. Am J Dis Child. 1979;133:632-5.

4. Brousse C, Nguyen-Plantin S, Friard S, Grenet D, Stern M. Étude de la densité minérale osseuse chez des patients insuffisants respiratoires chroniques. Rev Mal Respir. 2001;18(4 Pt 1):411-5.

5. Aris RM, Lester GE, Caminiti M, Blackwood AP, Hensler M, Lark RK, et al. Efficacy of alendronate in adults with cystic fibrosis with low bone density. Am J Respir Crit Care Med. 2004;169:7782.

6. Conway SP. Impact of lung inflammation on bone metabolism in adolescents with cystic fibrosis. Paediatr Respir Rev. 2001; 2:324-31.

7. Hecker TM, Aris RM. Management of osteoporosis in adults with cystic fibrosis. Drugs. 2004;64:133-47.

8. Schulze KJ, O'Brien KO, Germain-Lee EL, Baer DJ, Leonard AL, Rosenstein BJ. Endogenous fecal losses of calcium compromise calcium balance in pancreatic-insufficient girls with cystic fibrosis. J Pediatr. 2003;143:765-71.

9. Buntain HM, Greer RM, Schluter PJ, Wong JC, Batch JA, Potter JM, et al. Bone mineral density in Australian children, adolescents, and adults with cystic fibrosis: a controlled cross sectional study. Thorax. 2004;59:149-55.

10. Conway SP, Morton AM, Oldroyd B, Truscott JG, White H, Smith $\mathrm{AH}$, et al. Osteoporosis and osteopenia in adults and adolescents with cystic fibrosis: prevalence and associated factors. Thorax. 2000;55:798-804.

11. Campos LM, Liphaus BL, Silva CA, Pereira RM. Osteporose na infância e na adolescência. J Pediatr (Rio J). 2003;79:481-8.

12. Allgrove J. Use of biphosphonates in children and adolescents. J Pediatr Endocrinol Metab. 2002;15 Suppl 3:921-8.

13. U.S. Department of Health and Human Services, Center for Disease Control and Prevention, National Center for Health Statistics, CDC/NCHS/2000. Growth charts. Hyattsville. Disponível em: http://www.cdc.gov/growthcharts/. Acesso em: 20/08/2006. 
14. Slaughter MH, Lohman TG, Boilean RA, Horswill CA, Stillman RJ, Van Loan MD, et al. Skinfold equations for estimation of body fatness in children and youth. Hum Biol. 1988;60:709-23.

15. Anção MS, Cuppari L, Draibe AS, Sigulem D. Programa de apoio à Nutrição- NutWin-Versão 1,5 [CD- ROM]. São Paulo: Departamento de Informática em Saúde, SPDM- UNIFESP/ EPM; 2002.

16. Adde FV, Dolce P, Tanikawa CE, Uehara DY, Cardoso AL, Rosov T, et al. Suplementação dietética em pacientes com fibrose cística. J Ped 1997; 73 (5): 317-23.

17. Kerem E, Conway S, Elborn S, Heigerman H; Consensus Committee. Standards of care for patients with cystic fibrosis: a European Consensus 2005. J Cyst Fibros. 2005;4:7-26.

18. Frangolias DD, Paré PD, Kendle DL, Davidson AG, Wong L, Raboud $J$, et al. Role of exercise and nutrition status on bone mineral density in cystic fibrosis. J Cyst Fibros. 2003;2:163-70.

19. Reiter EO, Brugman SM, Pike JW, Pitt M, Dokoh S, Haussler MR, et al. Vitamin D metabolites in adolescents and young adults with cystic fibrosis: effects of sun and season. J Pediatr. 1985; 106:21-6.

20. Dia RM, Chauvet PR, Siqueira HR, Rufino R. Testes de função respiratória: do laboratório à aplicação com 100 exercícios para diagnósticos. São Paulo: Atheneu, 2000.

21. World Health Organization (WHO). Assessment of fracture risk and its application to screening for post-menopausal osteoporosis. Geneva: WHO; 1994. (WHO Technical Report Series n. 843).

22. EpiInfo 2000: a database and statistics program for public health professionals for use on Windows 95, 98, NT, and 2000 computers. Atlanta: Centers for Disease Control and Prevention; 2000.
23. Boucher RC. Human airway ion transport. Part one. Am J Resp Crit Care Med. 1994;150:271-81.

24. Boucher RC. Human airway ion transport. Part two. Am J Resp Crit Care Med. 1994;150:581-93.

25. Bhudhikanok GS, Lim J, Marcus R, Harkins A, Moss RB, Bachrach LK. Correlates of osteopenia in patients with cystic fibrosis. Pediatrics. 1996;97:103-11.

26. Buntain HM, Schluter PJ, Bell SC, Greer RM, Wong JC, Batch J, et al. Controlled longitudinal study of bone mass accrual in children and adolescents with cystic fibrosis. Thorax. 2006; 61:146-54.

27. Ujhelyi R, Treszl A, Vásárhelyi B, Holics K, Tóth M, Arató A, et al. Bone mineral density and bone acquisition in children and young adults with cystic fibrosis: a follow-up study. J Pediatr Gastroenterol Nutr. 2004;38:401-6.

28. Shane E, Silverberg SJ, Donovan D, Papadopoulos A, Staron RB, Adesso V, et al. Osteoporosis in lung transplantation candidates with end-stage pulmonary disease. Am J Med. 1996; 101:262-9.

29. Gronowitz E, Garemo M, Lindblad A, Mllström D, Strandvik B. Decreased bone mineral density in normal growing patients with cystic fibrosis. Acta Paediatr. 2003;92:688-93.

30. Littlewood JM. European cystic fibrosis society consensus on standards- a roadmap to "best care". J Cyst Fibros. 2005; 4:1-5.

31. Balfour-Lynn IM. Asthma in CF: corticosteroids. Pediatr Pulmonol. 2002; 34 Suppl 24:101-2.

Correspondência:

Reinaldo José do Amaral Caldeira

Avenida Rui Barbosa, 716

CEP 22250-020 - Rio de Janeiro, RJ

Tel.: (21) 2554.1719

E-mail: reinaldojacaldeira@yahoo.com.br 